\title{
CONHECIMENTO, ATITUDE E PRÁTICAS NA PREVENÇÃO DO CÂNCER DE COLO UTERINO E HPV EM ADOLESCENTES
}

\author{
Knowledge, attitude and practice in the prevention of cervical \\ cancer and HPV in adolescents \\ Conocimientos, actitudes y prácticas en la prevención de cáncer \\ cervicouterino y el VPH en adolescentes
}

Ana Luiza Vilela Borges ${ }^{3}$

\begin{abstract}
RESUMO
0 início sexual cada vez mais cedo propicia alta vulnerabilidade da adolescente a problemas da esfera sexual/reprodutiva, incluindo o câncer de colo uterino e a infecção pelo HPV. 0 presente estudo teve como objetivo identificar o conhecimento, atitude e prática na prevenção do câncer de colo uterino e infecção pelo HPV na população adolescente e avaliar as situações que as tornam vulneráveis. Trata-se de estudo transversal realizado em uma escola pública de São Paulo com 134 adolescentes entre 14 e 19 anos. Verificou-se idade de iniciação sexual aos 14,8 anos em média. Grande parte das adolescentes não apresentou conhecimento adequado sobre a prevenção desta neoplasia. A adesão ao Papanicolaou também se mostrou baixa. As estatísticas justificam a inserção da adolescente nos programas de detecção deste câncer. É preciso haver investimentos na educação sexual nas instituições de ensino e associar campanhas de Papanicolaou com atividades educativas, com enfoque adequado e linguagem apropriada.
\end{abstract}

Palavras-chave: Adolescente. Doenças Sexualmente Transmissíveis. Esfregaço Vaginal. Neoplasias do Colo do Útero.

\begin{abstract}
Sexual initiation increasingly early offers high vulnerability of adolescent to problems in the sexual/reproductive scenario, including cancer of the uterine cervix and HPV infection. This study aimed to identify the knowledge, attitude and practice in the prevention of cervical cancer and HPV infection in adolescent populations and assess the situations that make them vulnerable. The cross-sectional study was conducted in a public school in Sao Paulo with 134 adolescents between 14 and 19 years old. The age of sexual initiation is 14.8 years on average. Most adolescents had no adequate knowledge about the prevention of this malignancy. The access to Papanicolaou test was also low in this population. Statistics justify the inclusion of adolescents in programs for detection of cancer. We must invest in sex education in educational institutions and join the campaign for collecting Papanicolaou with educational activities with appropriate focus and language appropriate to change the current scenario.
\end{abstract}

keywords: Adolescent. Sexually Transmitted Diseases. Vaginal Smears. Uterine Cervical Neoplasms.

\section{Resumen}

La iniciación sexual cada vez más temprana propicia un nivel alto de vulnerabilidad de los adolescentes a los problemas de la esfera sexual / reproductiva, incluyendo el cáncer del cuello del útero y la infección por VPH. Este estudio tuvo como objetivo identificar los conocimientos, actitudes y prácticas en la prevención de cáncer cervical y la infección por VPH en la población adolescente y evaluar las situaciones que los tornan vulnerables. Este es un estudio transversal realizado en una escuela pública en Sao Paulo con 134 adolescentes entre 14 y 19 años. El promedio de la edad de iniciación sexual fue de 14,8 años. La mayoría de los adolescentes no tienen conocimientos adecuados sobre la prevención de este tipo de cáncer. También presentó bajas tasas de la prueba del Papanicolaou . Las estadísticas justifican la inclusión de estas adolescentes en los programas para la detección de este cáncer. Es indispensable invertir en la educación sexual en las instituciones educativas aunadas con campañas sobre la importancia del Papanicolaou vinculadas con actividades educativas que tengan un enfoque adecuado y un lenguaje apropiado.

Palabras clave: Adolescente. Enfermedades de Transmisión Sexual. Frotis Vaginal. Neoplasias del Cuello Uterino. 


\section{INTRODUÇ̃̃O}

A faixa etária mais acometida de câncer de colo uterino é entre 25 e 60 anos; entretanto, os adolescentes constituem uma população de alta vulnerabilidade para este agravo na medida em que o início da vida sexual os aproxima de problemas de saúde da esfera reprodutiva e sexual. ${ }^{1,2}$

Os adolescentes nem sempre usam métodos contraceptivos que os proteja contra gravidez indesejada e DST/AIDS na sua primeira relação sexual. ${ }^{3}$ Estudos revelam que o contágio pelo HPV (papiloma vírus humano), principal agente oncogênico do câncer de colo uterino, ocorre no início da vida sexual na adolescência ou por volta dos 20 anos. ${ }^{4-7}$

0 câncer de colo uterino é a neoplasia mais prevalente em mulheres com início precoce da atividade sexual e multiplicidade de parceiros sexuais. Outro fator relacionado ao aumento do risco para o desenvolvimento deste câncer é o uso de contraceptivos orais, as baixas condiç̧ões socioeconômicas e 0 uso irregular de preservativo.,

A incidência do HPV em adolescentes foi demonstrada em estudos, revelando taxa de $27 \%$; destas, $28,5 \%$ apresentaram na genotipagem molecular material genético viral de alto risco oncogênico. ${ }^{9}$

Em estudo retrospectivo realizado no Instituto Adolfo Lutz com revisão de 308.603 casos de câncer, de 1996 a 2001, verificou-se que a frequência de achados de atipias citológicas em esfregaços cervicovaginais vem crescendo gradativamente, sendo este aumento mais evidente entre as adolescentes, quando comparado ao aumento desses achados em mulheres adultas, o que justifica o rastreamento da neoplasia intraepitelial cervical (NIC) com a aplicação dos mesmos métodos utilizados para a mulher adulta jovem também na adolescência., 2,10

Embora as atipias citológicas em adolescentes sejam mais frequentemente de baixo grau, existe o risco de progressão para lesão de alto grau e carcinoma se não tratadas, especialmente se envolvem infecção por subtipos de HPV de alto risco. ${ }^{5}$

Um estudo realizado com 1.302 mulheres de 15 a 49 anos no sul do Brasil demonstrou que $97 \%$ já haviam tido relações sexuais, $70 \%$ tendo ocorrido antes dos 20 anos, e $57 \%$ nunca haviam realizado o exame para detecção precoce do câncer do colo do útero. Revelou-se que, quanto menor a idade, maior a probabilidade de não realização do exame. Além da menor idade, outros fatores condicionantes para a baixa adesão ao Papanicolaou foram: a cor parda ou preta, a precária inserção social (baixa escolaridade e pequeno valor de renda familiar) e a não presença do companheiro. ${ }^{11}$

Um estudo qualitativo realizado em um Centro de Saúde Escola com mulheres que estavam realizando o Papanicolaou pela primeira vez detectou que são muitos os motivos que as influenciam a não realizar o exame. Entre eles estão: desconhecimento do câncer de colo uterino, da técnica e da importância do exame preventivo, medo na realização do exame, medo de se deparar com resultado positivo para o câncer, sentimento de vergonha e constrangimento e, por fim, dificuldades para realizar o exame, sendo destacados tanto a dificuldade de acesso ao serviço quanto motivos relacionados ao papel da mulher no cuidado com a casa e os filhos. ${ }^{12}$

No Brasil, embora o Ministério da Saúde preconize desde 1998 a realização do exame para detecção precoce do câncer do colo uterino em todas as mulheres que já tiveram relações sexuais, com especial atenção àquelas com idade entre $25 \mathrm{e}$ 59 anos de idade, considera-se importante que os serviços de saúde ofereçam o acesso ao Papanicolaou à população adolescente. ${ }^{8}$

Diante do exposto, propôs-se o presente estudo, com o objetivo geral de identificar conhecimento, atitude e prática sobre a prevenção do câncer de colo uterino e infecção pelo HPV da população adolescente e avaliar as situações que as tornam vulneráveis, contribuindo para a melhoria do programa de prevenção e controle do câncer de colo do útero na cidade de São Paulo. Para tanto, busca analisar os fatores que condicionam a realização (ou não) do exame de Papanicolaou na população de 14 a 19 anos e descrever as características socioeconômicas, de saúde sexual e reprodutiva desta população.

\section{METODO}

Trata-se de um estudo transversal, realizado no período de março a maio de 2008, conduzido em uma escola pública do Distrito Administrativo de Cidade Ademar, região sul do município de São Paulo. Este distrito tinha uma população estimada de 244.934 habitantes em 2007, sendo $8,8 \%$ de adolescentes do sexo feminino de 10 a 19 anos. ${ }^{13}$ É um dos 96 distritos do município de São Paulo com piores índices de vulnerabilidade juvenil segundo a Fundação Seade. ${ }^{13}$

A escola possuía em março de 2008 um total de 763 alunos matriculados, 288 alunos entre a nona série do ensino fundamental e o terceiro ano do ensino médio. Desses, 152 eram do sexo feminino entre 14 e 19 anos. Os sujeitos da pesquisa foram constituídos por 134 adolescentes do sexo feminino, com idades entre 14 e 19 anos, estudantes da nona série do ensino fundamental ao terceiro ano do ensino médio. Dezoito alunas não participaram, sete por desautorização expressa dos pais, seis por não estarem presentes no momento da pesquisa e cinco por terem se recusado a participar.

Os dados foram coletados pela pesquisadora uma única vez, por meio de um formulário aplicado em local privativo contendo perguntas fechadas. 0 questionário autoaplicado dividiu-se em três partes: a primeira apresentava perguntas que objetivavam descrever as características sociodemográficas e econômicas das adolescentes e a segunda, questões que tratavam de suas características sexuais e reprodutivas; a terceira avaliava o conhecimento sobre o câncer de colo e infecção pelo HP e apresentava questões que buscavam identificar os fatores que dificultavam ou facilitavam a realização do Papanicolaou. 
0 conhecimento sobre o exame de Papanicolaou foi considerado adequado quando a adolescente sabia que o exame de Papanicolaou tem como objetivo o diagnóstico do câncer de colo uterino e que deve ser realizado anualmente e inadequado quando ela não soube responder sobre a finalidade do exame e/ou a frequência com que deve ser realizado.

Após a coleta dos dados, como contrapartida, foi fornecido para todas as alunas participantes ou não da pesquisa um material educativo sobre a prevenção do HPV e do câncer de colo uterino. Além disto, foi ministrada uma palestra sobre prevenção de câncer de colo uterino e HPV para uma plateia de aproximadamente 350 participantes entre adolescentes do sexo masculino e feminino.

\section{RESULTADOS}

Na distribuição etária verificou-se maior concentração na faixa etária de 16 e 17 anos (49,3\%), sendo quase a totalidade das adolescentes solteiras $(90,3 \%)$. Verifica-se que mais da
Os dados foram digitados e analisados por meio do Microsoft Excel 2007. Foi aplicado teste de diferença entre duas proporções pelo qui-quadrado no intuito de identificar as variáveis associadas à realização e conhecimento do Papanicolaou.

Todas as adolescentes matriculadas entre a nona série do ensino fundamental e o terceiro ano do ensino médio foram convidadas a participar da pesquisa. Os pais ou responsáveis pelas alunas menores de 18 anos foram informados sobre a pesquisa por meio de uma circular, com liberdade para manifestação contrária à participação de suas filhas na pesquisa. Foram adotados todos os procedimentos éticos na condução da pesquisa. 0 projeto foi submetido e aprovado por Comitê de Ética em Pesquisa, em conformidade com a Resolução CNS $n^{0}$ 196/96 sob protocolo 026/08.

metade dos pais não concluíram o ensino médio, sendo $56,7 \%$ dos pais e 67,9\% das mães. Quanto à renda familiar, 82,9\% referiram renda menor que seis salários mínimos mensais (Tabela 1).

Tabela 1: Distribuição das adolescentes de acordo com as características sociodemográficas. São Paulo, 2008.

\begin{tabular}{|c|c|c|}
\hline & f & $\operatorname{fr}(\%)$ \\
\hline \multicolumn{3}{|l|}{ Idade } \\
\hline $14-15$ ancs & 29 & 21,6 \\
\hline $16-17$ ancs & 66 & 49,3 \\
\hline $18-19$ ancos & 39 & 29,1 \\
\hline \multicolumn{3}{|l|}{ Média: 16,62} \\
\hline \multicolumn{3}{|l|}{ Mediana: 17} \\
\hline \multicolumn{3}{|l|}{ Desvio-padrão: 1,50} \\
\hline \multicolumn{3}{|c|}{ Valor máximo-minimo: 14-19 } \\
\hline \multicolumn{3}{|l|}{ Escolaridade do pai } \\
\hline Analfabeto & 7 & 5,2 \\
\hline Fundamental incompleto & 33 & 24,6 \\
\hline Fundamental completo & 19 & 14,2 \\
\hline Médio incompleto & 17 & 12,7 \\
\hline Médio completo & 33 & 24,6 \\
\hline Superior incompleto & 1 & 0,8 \\
\hline Superior completo & 7 & 5,2 \\
\hline Não soube informar & 17 & 12,7 \\
\hline \multicolumn{3}{|l|}{ Escolaridade da mầe } \\
\hline Analfabeto & 5 & 3,7 \\
\hline Fundamental incompleto & 49 & 36,6 \\
\hline Fundamental completo & 20 & 14,9 \\
\hline Médio incompleto & 17 & 12,7 \\
\hline Médio completo & 30 & 22,4 \\
\hline Superior incompleto & 6 & 4,5 \\
\hline Superior completo & 5 & 3,7 \\
\hline Năo soube informar & 2 & 1,5 \\
\hline \multicolumn{3}{|l|}{ Renda familiar* } \\
\hline 1-2 SM & 58 & 43,3 \\
\hline 3-5 SM & 53 & 39,6 \\
\hline $6-10 \mathrm{SM}$ & 20 & 14,9 \\
\hline Acima de $10 \mathrm{SM}$ & 3 & 2,2 \\
\hline \multicolumn{3}{|l|}{ Estado marital } \\
\hline Solteiras & 121 & 90,3 \\
\hline Unidas & 13 & 9,7 \\
\hline
\end{tabular}

* Valor do salário mínimo (SM) paulista na época da pesquisa: R \$ 410,00. 
0 perfil sexual e reprodutivo das 87 adolescentes com iniciação sexual (Tabela 2) mostra que $64,9 \%$ tiveram sua primeira relação em média aos 14,8 anos de idade (desviopadrão:1,4). 0 uso do preservativo masculino na iniciação sexual foi relatado por $78,2 \%$ delas, diminuindo o percentual para $59,7 \%$ quando questionadas sobre o uso do condom na última relação. A maioria $(97,0 \%)$ relatou uso do preservativo pelo menos uma vez na vida, e 46,0\% avaliaram que o uso da camisinha diminui o prazer.

Tabela 2: Perfil sexual e reprodutivo das adolescentes. São Paulo, 2008.

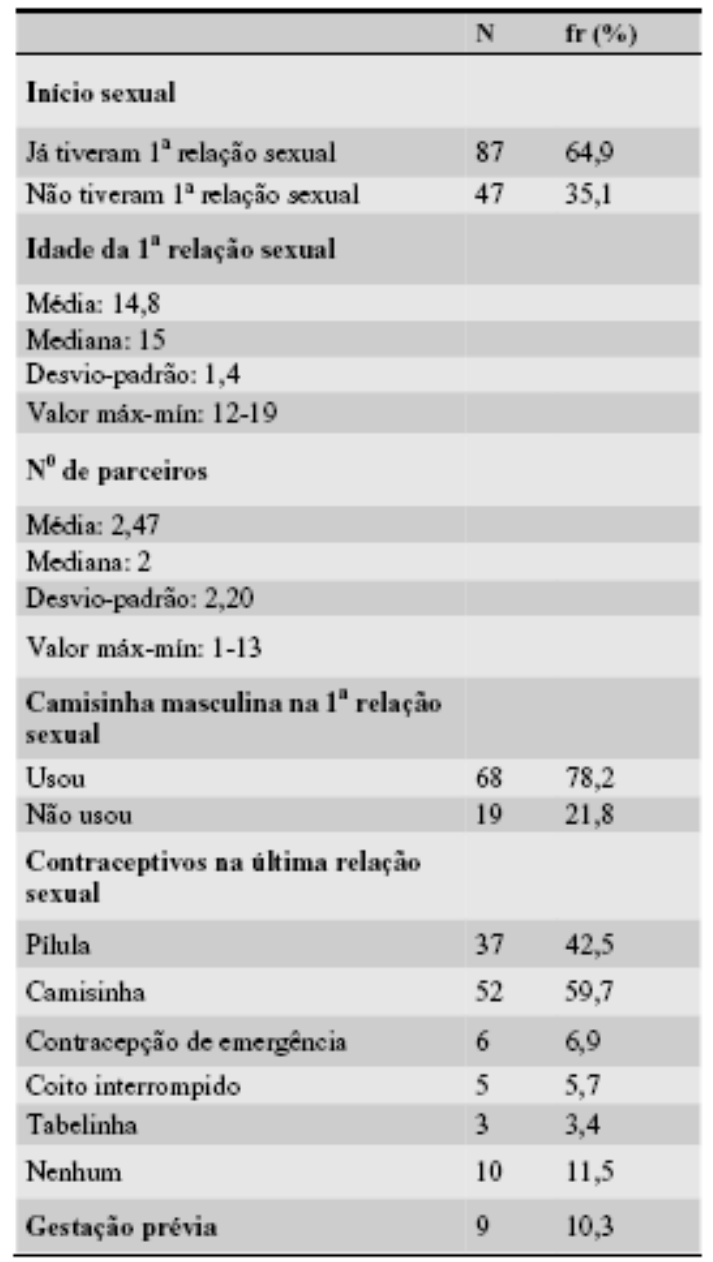

Para avaliar o conhecimento sobre a prevenção do câncer de colo uterino e a infecção pelo HPV, foram incluídas todas as 134 adolescentes participantes do estudo.

Quanto ao conhecimento, 67 (50,0\%) souberam que o objetivo desde exame é o seu diagnóstico, sendo observado menor percentual nas faixas etárias menores. Quando a variável analisada foi o estado marital, observou-se que as unidas apresentaram percentualmente maior conhecimento sobre 0 objetivo do Papanicolaou e sobre a periodicidade com que o preventivo deve ser realizado. Encontrou-se também maior percentual de respostas adequadas sobre o exame de filhas de pai e/ou mãe com nivel superior incompleto e menor percentual de corretas em filhas de mães analfabetas.

Quando avaliados o conhecimento e a variável renda familiar, constatou-se que as adolescentes que referiam renda acima de seis salários mínimos obtiveram melhores respostas quanto ao conhecimento sobre o objetivo do exame, quando comparadas àquelas que relataram menor renda $(p<0,01)$ (Tabela 3$)$. 
Tabela 3: Influência das variáveis idade, estado marital, escolaridade do pai e da mãe, renda familiar, início da vida sexual e realização do Papanicolaou no conhecimento sobre o objetivo do Papanicolaou, sobre o HPV como principal agente oncogênico e sobre a periodicidade com que o Papanicolaou deve ser realizado. São Paulo, 2008.

\begin{tabular}{|c|c|c|c|}
\hline \multirow[t]{2}{*}{ Variável } & \multicolumn{3}{|l|}{ Conhecimento } \\
\hline & Objetivo do Papa & HPV como causa do $\mathrm{Ca}$ & Frequência Papa \\
\hline \multicolumn{4}{|l|}{ Faixa etária } \\
\hline $\begin{array}{l}14-15 \text { anos } \\
16-17 \text { anos } \\
18-19 \text { anos }\end{array}$ & $\begin{array}{l}13(44,8 \%) \\
32(48,5 \%) \\
22(56,4 \%)\end{array}$ & $\begin{array}{l}4(15,3 \%) \\
11(16,7 \%) \\
11(28,2 \%)\end{array}$ & $\begin{array}{l}9(31,0 \%) \\
22(33,3 \%) \\
19(48,7 \%)\end{array}$ \\
\hline \multicolumn{4}{|l|}{ Estado marital } \\
\hline $\begin{array}{l}\text { Solteira }(n=121) \\
\text { Unidas }(n=13)\end{array}$ & $\begin{array}{l}59(48,8 \%) \\
9(69,2 \%)^{* *}\end{array}$ & $\begin{array}{l}23(19,0 \%) \\
2(15,4 \%)\end{array}$ & $\begin{array}{l}42(34,7 \%) \\
6(46,2 \%)\end{array}$ \\
\hline \multicolumn{4}{|l|}{ Escolaridade do pai } \\
\hline $\begin{array}{l}\text { Baixa escolaridade } \\
\text { Média escolaridade } \\
\text { Alta escolaridade } \\
\text { Não soube informar }\end{array}$ & $\begin{array}{l}27(47,8 \%) \\
24(48,0 \%) \\
5(62,5 \%) \\
9(52,9 \%)\end{array}$ & $\begin{array}{l}13(22,0 \%) \\
10(20,0 \%) \\
8(12,2 \%) \\
2(11,8 \%)\end{array}$ & $\begin{array}{l}19(32,2 \%) \\
20(40,0 \%) \\
4(50,0 \%) \\
5(29,4 \%)\end{array}$ \\
\hline \multicolumn{4}{|l|}{ Escolaridade da mãe } \\
\hline $\begin{array}{l}\text { Baixa escolaridade } \\
\text { Média escolaridade } \\
\text { Alta escolaridade } \\
\text { Não soube informar }\end{array}$ & $\begin{array}{l}33(44,6 \%) \\
21(44,7 \%)^{*} \\
9(81,8 \%)^{*} \\
1(50,0 \%)\end{array}$ & $\begin{array}{l}16(21,6 \%) \\
7(14,9 \%) \\
2(18,2 \%) \\
-\end{array}$ & $\begin{array}{l}25(33,8 \%) \\
18(38,3 \%) \\
5(45,5 \%) \\
-\end{array}$ \\
\hline \multicolumn{4}{|l|}{ Renda familiar } \\
\hline $\begin{array}{l}1-2 \mathrm{SM} \\
3-5 \mathrm{SM} \\
\text { Acima de } 6 \mathrm{SM}\end{array}$ & $\begin{array}{l}27(46,6 \%) \\
26(49.1 \%) \\
14(60,9 \%)^{*}\end{array}$ & $\begin{array}{l}12(20,7 \%) \\
7(13,2 \%) \\
5(21,7 \%)\end{array}$ & $\begin{array}{l}23(39,7 \%) \\
14(26,4 \%) \\
11(47,8 \%)\end{array}$ \\
\hline \multicolumn{4}{|l|}{ Inicio sexual } \\
\hline $\begin{array}{l}\text { Já tiveram } 1^{2} \text { relação } \\
\text { Nð̋o tiveram } 1^{2} \text { relação }\end{array}$ & $\begin{array}{l}48(55,2 \%)^{* *} \\
20(42,3 \%)\end{array}$ & $\begin{array}{l}24(27,6 \%)^{* *} \\
3(6,4 \%)\end{array}$ & $\begin{array}{l}39(44,8 \%)^{* *} \\
9(19,1 \%)\end{array}$ \\
\hline \multicolumn{4}{|l|}{$\begin{array}{l}\text { Realização do } \\
\text { Papanicolaou }\end{array}$} \\
\hline $\begin{array}{l}\text { Já fez Papa } \\
\text { Nunca fez Papa }\end{array}$ & $\begin{array}{l}26(56,5 \%)^{*} \\
38(43,2 \%)^{*}\end{array}$ & $\begin{array}{l}15(32,6 \%)^{* *} \\
13(14,8 \%)^{* *}\end{array}$ & $\begin{array}{l}23(50,0 \%) \\
25(28,4 \%)\end{array}$ \\
\hline \multicolumn{4}{|l|}{ Relato de DST prévia } \\
\hline $\begin{array}{l}\operatorname{Sim}(n=24) \\
\text { Nฮ̃。 }(n=63)\end{array}$ & $\begin{array}{l}11(45,8 \%) \\
59(53,2 \%) \\
\end{array}$ & $\begin{array}{l}3(12,5 \%) \\
22(19,8 \%)\end{array}$ & $\begin{array}{l}7(29,2 \%) \\
41(36,9 \%) \\
\end{array}$ \\
\hline
\end{tabular}

Das adolescentes que já haviam realizado o exame de Papanicolaou, 56,5\% conheciam seu objetivo, diferenciandose significantemente em relação àquelas que nunca realizaram o exame $(p<0,05)$ (Tabela 3).

A respeito do conhecimento sobre o HPV, verificou-se que $19,4 \%$ das adolescentes sabiam que o vírus é o principal agente oncogênico, aumentando para 32,6\% quando as adolescentes já haviam realizado o Papanicolaou, indicando uma diferença significativa $(p<0,01)$ com aquelas que nunca realizaram 0 exame (Tabela 3).

Analisando o conhecimento e a variável iniciação sexual, pode-se observar que as adolescentes que já tiveram a sua primeira relação sexual apresentaram maiores percentuais de respostas adequadas sobre prevenção do câncer de colo uterino e HPV quando comparadas às adolescentes "virgens" ( $p<$ 0,01) (Tabela 3).

Verificou-se que $17,9 \%$ das adolescentes referiram ter tido alguma doença sexualmente transmissível (DST), correspondendo a $27,6 \%$ das adolescentes com vida sexual ativa. As doenças relatadas foram: infecções vaginais por Candida sp ou Tricomonas SPem 20,7\% (18/87), infecções uretrais em 4,6\% (4/87), HPV em 2,3\% (2/87) e infecção por herpes vírus humano em $1,2 \%$.

Analisando as respostas dessas 24 adolescentes que relataram DST prévia, pode-se observar que: $21(87,5 \%)$ não sabiam que o câncer de colo uterino tem como principal agente oncogênico o HPV, 17 (70,8\%) não sabiam que o Papanicolaou deve ser realizado anualmente, e 13 (54,2\%) não sabiam que o preventivo tem como objetivo o diagnóstico do câncer de colo do útero. Estes percentuais são mais elevados quando comparados àquelas que não relataram DST prévia (Tabela 3).

Para avaliar o comportamento, atitude e práticas das adolescentes quanto à prevenção do câncer de colo uterino, foram excluídas aquelas que não tiveram iniciação sexual. Tal comportamento foi avaliado por meio das informações sobre fazer o Papanicolaou, ter feito o último exame há menos de um ano e manter regularidade da prevenção anual (Tabela 4). 
Tabela 4: Avaliação da atitude das adolescentes na prevenção do câncer de colo uterino segundo a faixa etária, estado marital, escolaridade do pai e da mãe e renda familiar. São Paulo, 2008.

\begin{tabular}{|c|c|c|c|c|c|c|}
\hline \multirow[b]{2}{*}{ Variável } & \multirow[b]{2}{*}{ n } & \multicolumn{5}{|l|}{ Atitude } \\
\hline & & $\begin{array}{l}\text { Nunca } \\
\text { fez Papa } \\
\mathrm{n}-41\end{array}$ & $\begin{array}{l}\text { Já } \\
\text { fez Papa } \\
\text { n-46 }\end{array}$ & $\begin{array}{l}\text { Último Papa a } \\
\text { menos de } 1 \text { ano } \\
\mathrm{n}-38\end{array}$ & $\begin{array}{l}\text { Faz Papa } \\
\text { anualmente } \\
n-26\end{array}$ & $\begin{array}{l}\text { Nâo faz Papa } \\
\text { anualmente } \\
n=20\end{array}$ \\
\hline \multicolumn{7}{|l|}{ Faixa etária } \\
\hline $14-15$ anos & $n-15$ & $9(60,0 \%)$ & $6(40,0 \%)$ & $6(100,0 \%)$ & $3(50,0 \%)$ & $3(50,0 \%)$ \\
\hline $16-17$ anos & $n-44$ & $23(52,3 \%)$ & $21(47,7 \%)$ & $17(81,0 \%)$ & $12(57,1 \%)$ & $9(42,9 \%)$ \\
\hline $18-19$ anos & $\mathrm{n}-28$ & $9(32,1 \%)$ & $19(67,9 \%)^{*}$ & $15(78,9 \%)$ & $11(57,9 \%)$ & $8(42,1 \%)$ \\
\hline \multicolumn{7}{|l|}{ Estade marital } \\
\hline Solteira & $\mathrm{n}-74$ & $39(52,7 \%)$ & $35(47,3 \%)$ & $29(82,9 \%)$ & $19(54,3 \%)$ & $16(45,7 \%)$ \\
\hline Unidas & $n-13$ & $2(15,3 \%)$ & $11(84,7 \%)^{*}$ & $9(81,8 \%)$ & $7(63,7 \%)$ & $4(36,3 \%)$ \\
\hline \multicolumn{7}{|l|}{ Escolaridade do pai } \\
\hline Baixa escolaridade & $n-42$ & $20(47,6 \%)$ & $22(52,4 \%)$ & $16(72,7 \%)$ & $12(54,5 \%)$ & $10(45,5 \%)$ \\
\hline Média escolaridade & $\mathrm{n}-36$ & $21(58,3 \%)$ & $15(41,7 \%)$ & $13(86,7 \%)$ & $7(39,5 \%)$ & $8(61,5 \%)$ \\
\hline Alta escolaridade & $n-4$ & $1(25,0 \%)$ & $3(75,0 \%)^{*}$ & $3(100,0 \%)$ & $2(66,7 \%)$ & $1(33,3 \%)$ \\
\hline Não soube informar & $n-8$ & $2(25,0 \%)$ & $6(75,0 \%)$ & $6(100,0 \%)$ & $5(83,3 \%)$ & $1(16,7 \%)$ \\
\hline \multicolumn{7}{|l|}{ Escolaridade da mãe } \\
\hline Baixa escolaridade & $n-47$ & $22(46,8 \%)$ & $26(53,2 \%)$ & $23(88,5 \%)$ & $16(61,5 \%)$ & $10(38,5 \%)$ \\
\hline Média escolaridade & $\mathrm{n}-33$ & $16(48,5 \%)$ & $17(51,5 \%)$ & $12(70,6 \%)$ & $9(52,9 \%)$ & $8(47,1 \%)$ \\
\hline Alta escolaridade & $\mathrm{n}-6$ & $3(50,6 \%)$ & $3(50,0 \%)^{*}$ & $3(100,0 \%)$ & $1(33,3 \%)^{* *}$ & $2(66,7 \%)$ \\
\hline Não soube informar & $\mathrm{n}-1$ & $1(100,0 \%)$ & - & - & - & - \\
\hline \multicolumn{7}{|l|}{ Renda familiar } \\
\hline $1-2 \mathrm{SM}$ & $n-41$ & $21(51,2 \%)$ & $20(48,8 \%)$ & $17(85,5 \%)$ & $13(65,0 \%)$ & $7(35,0 \%)$ \\
\hline $3-5 \mathrm{SM}$ & $\mathrm{n}-32$ & $13(40,6 \%)$ & $19(59,4 \%)$ & $14(43,7 \%)$ & $10(52,6 \%)$ & $9(47,4 \%)$ \\
\hline Acima de $6 \mathrm{SM}$ & $n-14$ & $7(50,0 \%)$ & $7(50,0 \%)$ & $7(100,0 \%)$ & $3(42,9 \%)$ & $4(57,1 \%)$ \\
\hline
\end{tabular}

${ }^{*} p<0,01 *^{*}<0,05$

Assim, das 87 que já tiveram iniciação sexual, 46 (52,9\%) fizeram o exame pelo menos uma vez. Quanto à frequência de realização, 26 (56,5\%) relataram realizá-lo anualmente e 28 $(60,8 \%)$ referiram achar o exame desconfortável.

Das 41 adolescentes que nunca fizeram o Papanicolaou, $26,8 \%$ não o fizeram por medo e $19,5 \%$ referiram como motivo vergonha.

As variáveis idade e estado marital exerceram influência positiva na atitude das adolescentes quanto à prevenção do câncer de colo uterino, sendo observado o maior percentual de realização de Papanicolaou na faixa etária de 18 a 19 anos $(67,9 \%)$ e entre aquelas com companheiro $(84,7 \%)$. Estas variáveis também exerceram influência positiva na atitude de realizar este exame com periodicidade anual.
Quanto às atitudes quanto à prevenção do câncer, em relação ao nível de escolaridade dos pais, provas estatísticas mostram que a variável escolaridade da mãe não exerceu influência positiva na atitude das filhas nem na realização do Papanicolaou ( $p<0,01)$, nem na periodicidade preconizada $(p<0,05)$. A escolaridade do pai influenciou positivamente a atitude das adolescentes na realização do exame $(p<0,01)$ e em ter realizado seu último Papanicolaou há menos de um ano. Já não houve influência sob a atitude de manter a periodicidade anual, assim como não foi comprovada a influência da variável renda sobre a atitude na prevenção do câncer de colo uterino.

\section{DISCUSSÃO}

Neste estudo encontrou-se média de idade de 14,8 anos da primeira relação, valores menores, porém próximos, aos 
encontrados em outros estudos. ${ }^{1,3}$ A tendência de antecipação da iniciação sexual traz maior preocupação, pois a imaturidade dos tecidos genitais é fator predisponente para o HPV e, consequentemente, para o câncer de colo uterino. ${ }^{14}$

Houve decréscimo no uso do preservativo masculino da última relação, quando comparada com a primeira relação sexual. 0 percentual de uso do condom na primeira relação encontrado neste estudo é relativamente baixo $(78,2 \%) \mathrm{em}$ relação a outro estudo realizado em São Paulo, ${ }^{3}$ cujo percentual encontrado foi de $93,7 \%$. Constatou-se que mais da metade das adolescentes não utilizou o preservativo na última relação sexual (59,7\%), e 46,0\% relataram que o uso do preservativo masculino diminui o prazer da relação. Estudo sugere que 0 condom vem sendo parcialmente substituído por outros métodos, a exemplo do contraceptivo hormonal. ${ }^{3} 0$ decréscimo no uso do preservativo também pode estar relacionado ao excesso de confiança nos parceiros. Esta justificativa é também observada em outras faixas etárias. ${ }^{15}$

Observou-se influência positiva da escolaridade dos pais em relação ao conhecimento sobre o objetivo do Papanicolaou, quando as adolescentes eram filhas de pais com nível superior. Além da escolaridade dos pais, outra variável que influenciou positivamente foi a renda familiar, mostrando maior conhecimento daquelas que declararam renda acima de seis salários mínimos. 0 fato de já terem tido a primeira relação sexual também exerceu influência positiva no conhecimento correto.

Um estudo aponta para uma alta vulnerabilidade das adolescentes ao HPV e atribuem esta vulnerabilidade à maior exposição da zona de transformação da cérvice (junção escamocolunar, JEC) durante a adolescência do que na vida adulta. É nesta zona que as células colunares podem sofrer metaplasia escamosa, sendo mais suscetível à infecção por agentes patogênicos de transmissão sexual, como o HPV, e é a área a partir da qual se origina a maior parte das lesões precursoras e carcinomas cervicais. Além disso, uma menor produção de muco cervical própria da fase de adolescência muco este que pode atuar como uma barreira protetora contra agentes infecciosos, associada a uma maior área de ectopia cervical - é um fator biológico de risco para a infecção pelo HPV em adolescentes. ${ }^{2}$

Apesar de alguns autores terem demonstrado alta incidência de infecção pelo HPV entre adolescentes, ${ }^{9,16}$ o presente estudo demonstrou que grande parte das adolescentes não tem conhecimento adequado sobre o câncer de colo uterino e sua prevenção, dado este que traz maiores preocupações quanto à saúde sexual e reprodutiva destas adolescentes.

Mesmo aquelas adolescentes que já realizaram o Papanicolaou desconhecem tanto o objetivo do exame quanto o HPV como principal agente oncogênico, mostrando uma deficiência das equipes de saúde, ou por não estarem promovendo educação em saúde no momento do exame ou por estarem utilizando técnicas de abordagem inadequadas para a faixa etária. Questiona-se se o treinamento desses profissionais contempla este novo público gerado pela iniciação sexual cada vez mais precoce.

A precocidade da prática sexual aproxima os adolescentes de problemas da esfera reprodutiva, ${ }^{4,7}$ o que se constata quando da observação de que 27,6\% tiveram história prévia de DST.

As adolescentes que relataram DSTs prévias apresentaram histórico de vulnerabilidade ao câncer de colo uterino mais alto que as adolescentes que não relataram DST prévia. 0 uso do preventivo, tanto na primeira quanto na última relação sexual, foi significativamente mais baixo nas adolescentes com DST prévia. 0 uso de contraceptivos orais, um dos fatores de risco para o desenvolvimento do câncer de colo uterino, ${ }^{8}$ foi mais elevado nestas adolescentes, assim como foi mais elevada a taxa de adolescentes que relataram não terem feito uso de nenhuma proteção contra DST e contra gestação indesejada na sua última relação sexual, o que reflete alto índice de gestação e de DST neste grupo. Além disso, o HPV, principal causa do câncer de colo uterino, estava presente no diagnóstico de 2,3\% dessas adolescentes.

As estatísticas de células precursoras do câncer de colo uterino em adolescentes e adultas jovens são alarmantes. Um estudo aponta uma taxa de incidência em adolescentes de lesões epiteliais de alto e baixo grau entre 6,9 e 8,4\%. ${ }^{10}$

Só no município de São Paulo, das 404 mulheres que morreram vítimas de neoplasia maligna de câncer de colo do útero no ano de 2005, 2,7\% tinham idade entre 20 e 29 anos. ${ }^{17}$ Sabendo-se que esta neoplasia tem uma evolução lenta, pode-se deduzir que muitas destas jovens adquiriram o câncer ainda na adolescência.

É senso comum que o esfregaço de Papanicolaou é o exame de rastreamento e detecção precoce das neoplasias cervicais e de células precursoras do câncer de colo do útero. É possível diagnosticar lesões ainda na fase intraepitelial (não invasiva) em mulheres assintomáticas dada a lenta evolução deste câncer.

Ainda se evidenciam muitos mitos, preconceitos e fantasias envolvendo a sexualidade. 0 baixo acesso ao conhecimento sobre as prevenções do câncer de colo uterino e sexualidade no convívio familiar, principalmente em adolescentes de baixa renda, deve ser compensado pela informação na sala de aula e em campanhas de educação em saúde, utilizando-se de técnicas e linguagens apropriadas para esta população.

Ao avaliar a atitude, observou-se também que quase metade $(47,1 \%)$ das adolescentes que têm vida sexual ativa não havia feito o exame de Papanicolaou, índice considerado alto quando comparado aos estudos realizados em São Paulo com uma amostra representativa da população entre 15 e 49 anos, que demonstrou que apenas $13,8 \%$ das mulheres nunca haviam feito o teste na vida. ${ }^{18}$ Há necessidade de ampliar o acesso da população adolescente no sentido de obter uma maior adesão dessas mulheres à coleta do Papanicolaou, aumentando a detecção de células precursoras do câncer e tratamento precoce, diminuindo, consequentemente, a mortalidade por causas relacionadas. 
A precária inserção social (baixa escolaridade e pequeno valor de renda familiar) é um fator associado à baixa adesão ao Papanicolaou. ${ }^{11}$ Neste estudo não se observou influência da renda familiar na atitude das adolescentes; porém, é bom lembrar que o estudo tomou como sujeitos estudantes de uma instituição pública, localizada em um dos distritos com piores índices de vulnerabilidade social, ${ }^{13}$ e que a variação de renda familiar observada foi pequena.

Estas adolescentes apresentaram várias razões para justificar a não realização do exame preventivo, sendo o medo e a vergonha os principais motivos relatados: "Fazer o exame é embaraçoso, desconfortável, tenho vergonha e medo"; estes motivos são referidos por $32,5 \%$ das entrevistadas em outros estudos. ${ }^{18}$ Diante disso, é possível pensar na dificuldade de acesso destas adolescentes não só ao exame propriamente, mas aos serviços de saúde em geral.

Detectou-se neste estudo expressiva necessidade por informação pela população, dado que $31,4 \%$ das adolescentes

\section{CONCLUSÃO}

Apesar das iniciativas em termos de políticas de saúde voltadas à saúde da mulher, como as campanhas de conscientização e divulgação para realização do Papanicolaou, com acesso facilitado e gratuito, considera-se que este é um grupo altamente vulnerável às DSTs e ao câncer de colo de útero.

São necessários investimentos no desenvolvimento de práticas de promoção à saúde para modificar este quadro. Nesse sentido, é preciso que seja revista a educação sexual nas

\section{REFERÊNCIAS}

1. Silva P, Oliveira MDS, Matos MA, Tavares VR, Medeiros M, Brunini S, et al. Comportamento de risco para as doenças sexualmente transmissíveis em adolescentes escolares de baixa renda. Rev Eletr Enferm 2005; 7 (2): 185-89.

2. Longatto Filho A, Etlinger D, Gomes NS, Cruz SV, Cavalieri MJ. Frequência de esfregaços cérvico-vaginais anormais em adolescentes e adultas: revisão de 308.630 casos. Rev Inst Adolfo Lutz 2003; 62 (1): 31-34.

3. Borges A, Schor N. Início da vida sexual na adolescência e relações de gênero: um estudo transversal em São Paulo, Brasil, 2002. Cad Saude Publica 2005 mar/abr; 21 (2): 499-507.

4. Ministério da Saúde (BR). Instituto Nacional de Câncer - INCA. Câncer de colo do útero. [on-line] [citado 19 jan 2008]. Disponível em: http://www.inca.gov.br/

5. Walboomers JMM, Jacobs MV, Manos MM, Bosch FX, Kummer JA, Shah KV, et al. Human papillomavirus, a necessary cause of invasive cervical cancer wordwide. J Pathol 1999 Sep; 189: 12-9.

6. Guanilo MCTU, Moura RF, Conceição CA, Nichiata LYI. Papilomavírus humano e neoplasia cervical: a produção científica dos países da América Latina e Caribe nos últimos 11 anos. DVST- J Bras Doenças Sex Transm 2006 out; 18 (1): 58-61. identificaram a falta de conhecimento como motivo principal da não adesão ao exame preventivo.

Porém, o que mais chamou a atenção foi o fato de que, das adolescentes que já tiveram sua primeira relação sexual, quase um terço relatou DST prévia. E foram nestas em que se observou maior vulnerabilidade ao câncer de colo uterino, com uma menor adesão ao uso da camisinha, tanto na sexarca quanto na última relação, além de uma baixa adesão ao Papanicolaou e maior média de parceiros sexuais. Foi também este grupo a apresentar maior taxa de gravidez.

0 estudo, ao identificar características comportamentais relativas aos conhecimentos e atitudes sobre a prevenção do câncer de colo uterino e infecção pelo HPV da população adolescente e avaliar as situações que a tornam vulneráveis, indica a necessidade de aprofundamento da temática, contribuindo com o debate e a intervenção em torno da saúde desta população.

instituições de ensino para que essas ofereçam suporte educacional em saúde para estas jovens. Também é preciso associar às campanhas de coleta de Papanicolaou atividades educativas com o enfoque adequado a cada faixa etária, e com uma linguagem direita e apropriada, quebrando mitos e desmistificando tabus.

É preditivo que se invista também em novas pesquisas sobre a sexualidade e saúde reprodutiva desta população, analisando as influências sociais, econômicas e culturais e, principalmente, as questões de gênero que perpassam todas elas.

7. Ferenczy A. Epidemiology and clinical pathohysiology of condylomata acuminata. Am J Obstet Gynecol 1995 Apr; 172: 1331-39.

8. Ministério da Saúde (BR). Secretaria de Atenção à Saúde. Instituto Nacional de Câncer-INCA. Coordenação de Prevenção e Vigilância. Estimativa 2008: incidência de câncer no Brasil. Rio de Janeiro (RJ); 2007.

9. Barros LDF. Infecção genital pelo papiloma vírus humano (HPV) em adolescentes - diagnóstico biomolecular [tese de doutorado]. Maceió (AL): Universidade Federal de Alagoas; 2006.

10. Monteiro DLM. A cérvice uterina da adolescente: estudo da prevalência e dos fatores associados ao câncer de colo uterino e suas lesões precursoras em população de adolescentes atendidas em Hospital Público do Município do Rio de Janeiro [tese de doutorado]. Rio de Janeiro (RJ): Instituto Fernandes Figueira / FIOCRUZ; 2004.

11. Cesar AJ, Horta BL, Gomes G, Houlthause RS, Willrich RM, Kaercher $A$, et al. Fatores associados a não realização de exame citopatológico de colo uterino no extremo sul do Brasil. Cad Saude Publica 2003 set/ out; 19 (5): 1365-372.

12. Ferreira MLSM. Motivos que influenciam a não-realização do exame de papanicolaou segundo a percepção de mulheres. Esc Anna Nery Rev Enferm 2009 abr/jun; 13 (2): 378-84. 
13. Secretaria de Economia e Planejamento (SP). Fundação Sistema Estadual de Análise de Dados - SEADE. Índice de vunerabilidade juvenil. .[on-line] [citado 15 jan 2008]. Disponível em: http:// www.seade.gov.br/produtos/ivj/.

14. Conti FS, Bortolin S, Külkamp IC. Educação e promoção à saúde: comportamento e conhecimento de adolescentes de colégio público e particular em relação ao papilomavírus humano. DVST- J Bras Doenças Sex Transm 2006 out; 18 (1): 30-35.

15. Maia C, Guilhem D, Freitas D. Vulnerabilidade ao HIV/AIDS de pessoas heterossexuais casadas ou em união estável. Rev Saude Publica 2008 abr; 42 (2): 242-48.
16. Taquette SR, Andrade RB, Vilhena MM, De Paula MC. A relação entre as características sociais e comportamentais da adolescente e as doenças sexualmente transmissíveis. Rev Assoc Med Bras 2005 maio/ jun; 51(3): 148-52.

17. Ministério da Saúde (BR). DATASUS: indicadores de mortalidade. Taxa de mortalidade específica por neoplasias malignas. [citado 07 set 2008]. Disponível em: http://tabnet.datasus.gov.br/

18. Pinho AA, França Junior I, Schraiber LB, D'Oliveira AFPL. Cobertura e motivos para a realização ou não do Teste de Papanicolaou no município de São Paulo. Cad Saude Publica 2003; 19 (sup 2): S303S313. 